\title{
Effect of Maternal Labor and Mode of Delivery on Polymorphonuclear Leukocyte Function in Healthy Neonates
}

\author{
SHAISTA S. USMANI, SHAHID KAMRAN, RITA G. HARPER, RAUL A. WAPNIR, AND \\ RAJEEV MEHTA \\ Department of Pediatrics, Division of Perinatal Medicine, and Department of Obstetrics and Gynecology, North \\ Shore University Hospital-Cornell University Medical College, Manhasset, New York 11030
}

\begin{abstract}
In this study, effect of maternal labor and mode of delivery on polymorphonuclear leukocyte (PMN) chemiluminescence and random and chemotactic motility was evaluated in healthy full-term neonates. PMN were obtained from cord blood of three groups of neonates: group I, 24 vaginally delivered neonates; group II, 22 neonates delivered by elective cesarean section without labor; and group III, 18 neonates delivered by cesarean section after labor. In group III, six neonates were delivered by cesarean section for fetal distress with acidemia and 12 for failure of progression of labor. Peak chemiluminescence of PMN in group III was depressed compared with groups I and II $(p<0.01)$. There was no difference in the peak chemiluminescence of PMN from neonates in group I versus group II. Random motility of PMN in group III was increased compared with the random motility in groups I and II ( $p$ $<0.05$ ). Chemotactic motility of PMN was comparable in all three groups. In group III, a negative correlation was noted between peak chemiluminescence of PMN and the duration of labor $(p<0.001)$, whereas no such correlation was observed in group I despite a similar duration of labor. There was no correlation between duration of labor and random and chemotactic motility of PMN in groups $I$ and III. The results of this study indicate that labor and mode of delivery per se have no effect on PMN function and that factors other than labor such as fetal acidemia, fetal distress, arrested labor, or maternal administration of drugs may play a role in alteration of PMN function. (Pediatr Res 33: 466-468, 1993)
\end{abstract}

\section{Abbreviations}

PMN, polymorphonuclear leukocyte $\mathrm{CW} / \mathrm{L}$, cesarean section with labor

PMN function as the first line of defense against a variety of microbial pathogens. Previous studies have demonstrated that a number of developmental deficiencies exist in the phagocytic and intracellular killing ability of neonatal PMN as compared with adult PMN. These PMN defects may make the human neonate susceptible to infection $(1-5)$.

PMN function in the neonate has been reported to be affected by various methods of delivery. Kinoshita et al. (6) demonstrated that cord blood PMN adherence was enhanced in neonates

Received October 6, 1992; accepted December 30, 1992.

Correspondence and reprint requests: Shaista S. Usmani, M.D., Associate Neonatologist, Division of Perinatal Medicine, North Shore University Hospital, 300 Community Dr., Manhasset, NY 11030. delivered vaginally and by cesarean section after labor compared with neonates delivered by cesarean section without labor. Bruce et al. (7) noted that spontaneous expression of CR 1 (complement receptors believed to be necessary for optimal phagocytosis) was greater in neonates delivered vaginally than in infants delivered by cesarean section without labor. The authors suggested that the increase in receptor expression may be due to factors associated with labor (7).

Frazier et al. (8) demonstrated that oxidative-metabolic leukocyte functions were significantly lower in cord blood PMN from neonates delivered vaginally and by $\mathrm{CW} / \mathrm{L}$ than in cord blood PMN from infants delivered by cesarean section without labor. The cause of the difference in PMN function of infants delivered after labor remains unknown. Significant differences have been demonstrated in corticosteroid, fatty acid, and prostaglandin concentrations in neonates delivered after labor compared with neonates delivered by cesarean section without labor (9-11). The role of maternal labor and mode of delivery on PMN function has not been resolved. The purpose of this study was to determine whether: 1) the mode of delivery has an effect on chemiluminescence, random motility, and chemotaxis of PMN obtained from cord blood of full-term healthy neonates; 2) there is a correlation between altered PMN function and duration of labor; and 3) various perinatal factors have an effect on PMN functions.

\section{MATERIALS AND METHODS}

Study population. The study population consisted of three groups of healthy full-term neonates born to women with normal medical and obstetric histories at North Shore University Hospital.

Group I comprised 24 vaginally delivered neonates (mean \pm SD gestational age, $39.7 \pm 0.96 \mathrm{wk}$; birth weight, $3379 \pm 438 \mathrm{~g}$ ). There were 13 males and 11 females. Fifteen women received epidural anesthesia (bupivacaine with or without epinephrine), seven women received local lidocaine anesthesia, and two received no anesthetics. There was no other drug used in these women.

Group II comprised 22 neonates delivered by elective cesarean section without labor (mean $\pm \mathrm{SD}$ gestational age, $39.5 \pm 1.05$ wk; birth weight, $3435 \pm 470 \mathrm{~g})$. There were seven males and 15 females. Epidural anesthesia (bupivacaine with epinephrine) was administered to all mothers in this group.

Group III comprised 18 neonates delivered by cesarean section after labor (mean $\pm \mathrm{SD}$ gestational age, $40.0 \pm 1.04 \mathrm{wk}$; birth weight, $3650 \pm 486 \mathrm{~g}$ ). There were 13 males and five females. Labor was induced by prostaglandin $\mathrm{E}_{2}$ suppository in three women and augmented with oxytocin in 11 women. All cesarean sections were performed under epidural (bupivacaine with epi66 
an emergency cesarean section because of fetal heart rate deceleration with fetal acidemia $(\mathrm{pH}<7.15)$. Twelve infants were delivered because of failure of progression of labor.

Exclusion criteria included neonates born to mothers with $>48$ h rupture of membranes, maternal fever (temperature $>38^{\circ} \mathrm{C}$ ), and mothers receiving antibiotics and/or other drugs. Neonates who subsequently became sick while in the nursery were also excluded from the study. The study was approved by the Institutional Research and Publications Review Committee in human subjects at North Shore University Hospital. Informed consent is not required for obtaining cord blood from human subjects by the Institutional Research and Publications Review Committee.

Collection of blood. In the delivery room, cord blood was obtained from the veins on the fetal side of the placenta after the placenta had been extracted by the obstetrician and placed in a sterile dish.

Blood was obtained using an 18-gauge needle and a $10-\mathrm{mL}$ plastic syringe. After collection, blood was heparinized with 10 units/mL of heparin and PMN function studies were performed within half an hour of collection.

$P M N$ function studies. PMN were separated according to the method of Boyum (12) and washed twice in Hanks' balanced salt solution without $\mathrm{Ca}^{++}, \mathrm{Mg}^{++}$, and phenol red. After separation, cell suspension contained 85\% PMN and trypan blue dye showed $>95 \%$ PMN viability. Cells were adjusted to a concentration of $1.0 \times 10^{6} \mathrm{PMN} / \mathrm{mL}$ in Hanks' balanced salt solution for chemiluminescence and $2.0 \times 10^{6} \mathrm{PMN} / \mathrm{mL}$ in $2 \% \mathrm{BSA}$ for random and chemotactic motility.

Luminol-enhanced (Sigma Diagnostics, St. Louis, MO) chemiluminescence, an assay of oxidative and metabolic function of phagocytizing PMN, was measured in a liquid scintillation system (Beckman model LS-230, Beckman Instruments, Inc., Fullerton, CA) using latex particles $0.8 \mu \mathrm{m}$ in diameter (Seragon Diagnostics, Indianapolis, IN) as a stimulant (13-15). Samples were tested in duplicate, and levels of chemiluminescence in cpm $\left(\mathrm{cpm} \times 10^{3}\right)$ for $1.0 \times 10^{6} \mathrm{PMN}$ were recorded every $30 \mathrm{~s}$ for a 30-min period.

Random and chemotactic motility was tested by modified Boyden technique using blind well chambers (Neuro-Probe, Inc., Bethesda, MD) and 5- $\mu \mathrm{m}$ pore cellulose nitrate filters (Millipore Corp., Bedford, MA) (16). Tissue culture medium-199 (Gibco Laboratories, Grand Island, NY) was used to determine random motility. Pooled human adult serum (10\%) activated by $1 \mathrm{mg} /$ $\mathrm{mL}$ zymosan (Sigma Laboratories, St. Louis, MO) was used as a chemoattractant. Adult serum was used to correct the deficiency of neonatal serum factors.

Samples were tested in duplicate after incubation of PMN for $2 \mathrm{~h}$ at $37^{\circ} \mathrm{C}$. Random motility and chemotaxis were expressed as the mean depth in $\mu \mathrm{m}$ at which the leading front of PMN was in focus using $a \times 40$ objective. Mean value represents the average of readings in five randomly chosen microscopic fields in each filter.

Statistical analysis. Analysis of variance followed by Tukey's test was used to assess the difference between means of each of the PMN functions for the three modes of delivery and labor groups (i.e. vaginal delivery, $\mathrm{CW} / \mathrm{L}$, and cesarean section without labor). Pearson product-moment correlation coefficient was used to determine the correlation between number of hours of labor and each of the PMN functions. $\chi^{2}$ test was used to evaluate the relationship of fetal distress and PMN functions. A value less than or equal to 0.05 was considered statistically significant.

\section{RESULTS}

Intergroup comparison. There was no significant difference in maternal age, race, parity, or duration of gestation among the three groups. The mean duration of labor was similar in group I (vaginal delivery) and group III (cesarean section after labor). Birth weight and Apgar scores of all infants in the three groups were comparable.
In group I (vaginal delivery) and group II (cesarean section without labor), there were no neonates with a history of fetal distress, failure of progression of labor, or maternal use of drugs except for the use of epidural and/or local anesthetics. In group III (CW/L), six neonates were delivered by an emergency cesarean section for fetal distress and the remaining 12 neonates for failure of progression of labor.

$P M N$ functions. Peak chemiluminescence activity of cord blood PMN from neonates in group III $(\mathrm{CW} / \mathrm{L})$ was significantly depressed compared with the other two groups $(p<0.01$, Table 1). Peak chemiluminescent activity was delayed in onset and the subsequent decline in activity was more rapid for group III neonates. There was no difference in the peak chemiluminescence values or kinetics of the chemiluminescence of PMN from neonates in group I (vaginal delivery) versus neonates in group II (cesarean section without labor).

Random motility of PMN from neonates in group III (CW/ L) was significantly increased compared with the other two groups $(p<0.05$, Table 1$)$. Chemotactic motility of cord PMN in response to zymosan-activated serum was comparable in all three groups (Table 1).

PMN functions and duration of labor. In group III neonates $(\mathrm{CW} / \mathrm{L})$, a negative correlation was noted between the peak chemiluminescence values of PMN and the duration of labor ( $p$ $<0.001$, Fig. 1). The kinetics of the chemiluminescence response in group III neonates also differed from group I neonates in that the time to reach peak chemiluminescence was increased as the duration of labor lengthened ( $p<0.01$, Fig. 2). In group I neonates (vaginal delivery), there was no correlation between the peak chemiluminescence values of PMN or the time to reach the peak chemiluminescence and the duration of labor despite similar duration of labor in group I versus group III (Figs. 1 and 2).

When random and chemotactic motility of PMN from neonates in group I (vaginal delivery) and group III $(\mathrm{CW} / \mathrm{L})$ were

Table 1. Comparison of PMN functions in full-term neonates delivered by vaginal route (group I), cesarean section without labor (group II), and cesarean section with labor (group III)*

\begin{tabular}{lccc}
\hline \multicolumn{1}{c}{ Test variables } & $\begin{array}{c}\text { Group I } \\
(n=24)\end{array}$ & $\begin{array}{c}\text { Group II } \\
(n=22)\end{array}$ & $\begin{array}{c}\text { Group III } \\
(n=18)\end{array}$ \\
\hline $\begin{array}{l}\text { Chemiluminescence } \\
\left(\mathrm{cpm} \times 10^{3}\right)\end{array}$ & $443 \pm 72$ & $460 \pm 99$ & $278 \pm 68 \dagger$ \\
$\begin{array}{c}\text { Random motility } \\
(\text { depth in } \mu \text { m) }\end{array}$ & $44.7 \pm 13.5$ & $47 \pm 13.8$ & $58.6 \pm 13.6 \ddagger$ \\
$\begin{array}{l}\text { Chemotaxis (depth in } \\
\mu \mathrm{m})\end{array}$ & $71.8 \pm 16.0$ & $63.0 \pm 16.9$ & $68.3 \pm 15.8$ \\
\end{tabular}

* Values represent mean $\pm \mathrm{SD}$.

$\dagger p<0.01$, group III $v s$ groups I and II.

$\ddagger p<0.05$, group III $v s$ groups I and II.

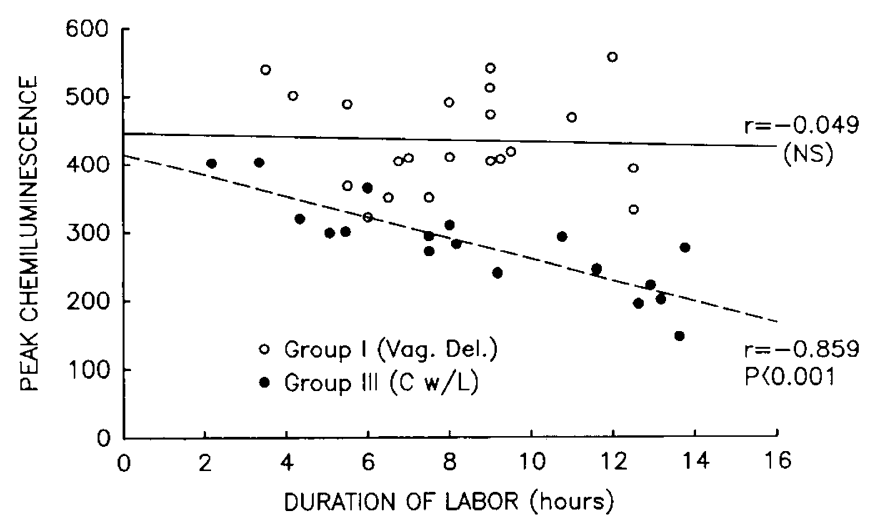

Fig. 1. Relationship of duration of labor and PMN peak chemiluminescence values in neonates in group I (vaginal delivery, open circles and solid line $)$ and group III (CW/L, filled circles and broken line). 


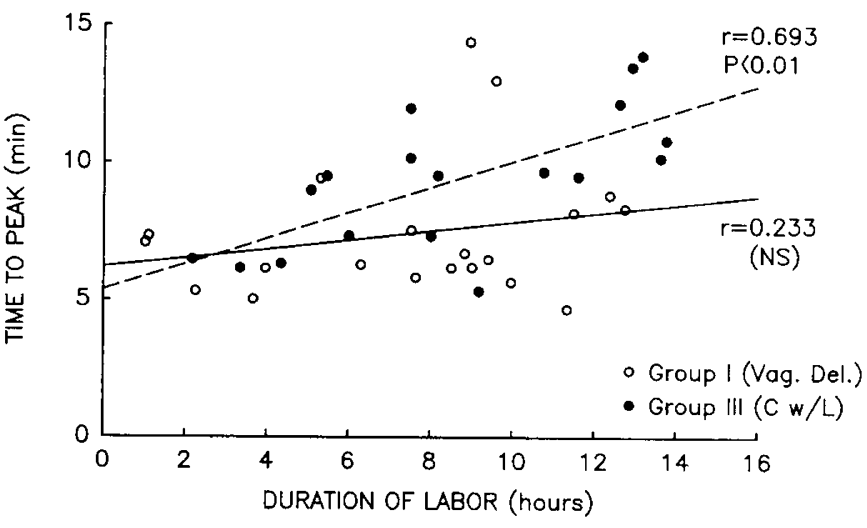

Fig. 2. Relationship of duration of labor and time to reach peak chemiluminescence by PMN in neonates in group I (vaginal delivery, open circles and solid) and group III (CW/L, filled circles and broken line).

compared as to the duration of labor, no correlation was noted in spontaneous or stimulated PMN motility and the duration of labor.

PMN functions and other perinatal factors. In group III (CW/ L), six neonates were delivered because of fetal distress with acidemia and 12 for failure of progression of labor. Labor was induced by prostaglandin $\mathrm{E}_{2}$ suppository in two women experiencing fetal distress and one woman with arrested labor. Oxytocin was used to augment labor in three women with fetal distress and eight women with failure of progression of labor. PMN chemiluminescence in six neonates with fetal distress was lower than the chemiluminescence values of 12 neonates with arrested labor $\left(247 \pm 66\right.$ versus $293 \pm 68 \mathrm{cpm} \times 10^{3}$, mean \pm SD) but did not reach statistical significance. Similarly, random and chemotactic motility of PMN $(59 \pm 12.6$ and $74 \pm 11.6 \mu \mathrm{m}$, mean $\pm \mathrm{SD}$ ) from neonates with fetal distress did not differ from random and chemotactic motility $(60 \pm 10.3$ and $68.5 \pm 9.0 \mu \mathrm{m}$, mean $\pm \mathrm{SD}$ ) of those with failure of progression of labor.

There were no neonates with a history of fetal distress, acidemia, failure of progression of labor, or maternal use of prostaglandin $\mathrm{E}_{2}$ and Pitocin in groups I and II.

\section{DISCUSSION}

This study indicates that PMN chemiluminescence was depressed and the peak chemiluminescence response was delayed in neonates delivered by cesarean section after experiencing labor (group III). Cesarean section was performed in these women because of the occurrence of fetal distress or failure of progression of labor, both of which may impair fetoplacental perfusion and cellular metabolism. This result is not surprising, inasmuch as fetal hypoxia and failure of progression of labor have been associated with increased risk of sepsis, particularly early onset disease (17).

Postnatally, neonates who have been stressed have also demonstrated depressed PMN chemiluminescence and oxidative metabolic responses $(18,19)$. Wright et al. $(20)$ found that PMN from stressed neonates with a variety of infectious and noninfectious illnesses had depressed bactericidal activity when compared with healthy controls. Frazier et al. (8) noted that zymosanstimulated oxygen consumption, hexose monophosphate shunt activity, and nitroblue tetrazolium dye reduction were significantly lower in cord PMN from five neonates delivered by $\mathrm{CW} /$ $\mathrm{L}$ and 15 neonates delivered vaginally than in cord PMN from
15 neonates delivered by cesarean section without labor. These authors did not mention whether there were any neonates with fetal acidemia or fetal distress in the vaginally delivered group or the reason for cesarean section after labor. In the present study, the depressed chemiluminescence response seen only in neonates delivered by $\mathrm{CW} / \mathrm{L}$ suggests that factors other than labor per se (such as fetal acidemia, fetal distress, arrested labor, or maternal administration of drugs) may play a role in alteration of the PMN function.

Random motility of PMN from neonates in group III (CW/ L) was enhanced $(p<0.05)$ compared with that of neonates in groups I and II (vaginal delivery and cesarean section without labor). The cause for the enhanced random motility in group III neonates is not entirely clear. It may be that fetal acidemia, use of prostaglandins, or factors associated with arrested labor may affect PMN cell membrane depolarization and/or the number of microtubules and thereby influence random motility (1-5).

In summary, results of this study indicate that labor or mode of delivery per se may have no effect on PMN function but that fetoplacental perfusion and/or alteration in metabolism may alter PMN function in neonates.

\section{REFERENCES}

1. Miller ME 1979 Phagocyte function in the neonate: selected aspects. Pediatrics 64:709-712

2. Quie PG, Mills EL 1979 Bactericidal and metabolic function of polymorphonuclear leukocytes. Pediatrics 64:719-721

3. Wilson CB 1986 Immunologic basis for increased susceptibility of the neonate to infection. J Pediatr 108:1-12

4. Hill HR 1987 Biochemical, structural and functional abnormalities of polymorphonuclear leukocytes in the neonate. Pediatr Res 22:375-382

5. Yang KD, Hill HR 1991 Neutrophil function disorders: pathophysiology, prevention and therapy. J Pediatr 119:343-354

6. Kinoshita Y, Masuda K, Kobayashi Y 1991 Adherence of cord blood neutrophils: effect of mode of delivery. J Pediatr 118:115-117

7. Bruce MC, Baley JE, Medvik KA, Berger M 1987 Impaired surface membrane expression of $\mathrm{C} 3 \mathrm{bi}$ but not $\mathrm{C} 3 \mathrm{~b}$ receptors on neonatal neutrophils. Pediatr Res 21:306-311

8. Frazier JP, Cleary TG, Pickering LK, Kohl S, Ross PJ 1982 Leukocyte function in healthy neonates following vaginal and cesarean section deliveries. J Pediatr 101:269-272

9. Cawson MJ, Anderson ABM, Turnbull AC 1974 Cortisol, cortisone, and IIdeoxycortisol levels in human umbilical and maternal plasma in relation to the onset of labor. J Obstet Gynaecol Br Commonw 81:737-745

10. Murphy BEP 1973 Does human fetal adrenal play a role in parturition? Am J Obstet Gynecol 115:521-525

11. Karim SMM, Devlin J 1967 Prostaglandin content of amniotic fluid during pregnancy and labor. J Obstet Gynaecol Br Commonw 74:230-234

12. Boyum A 1968 Separation of leukocytes from blood and bone marrow. Scand J Clin Lab Invest 21:7-12

13. Steven P, Winston DJ, Van Dyke K 1978 In vitro evaluation of opsonic and cellular granulocyte function by luminal enhanced chemiluminescence: utility in patients with severe neutropenia and cellular deficiency states. Infect Immunol 22:41-51

14. Welch WD 1980 Correlation between measurement of luminol dependent chemiluminescence response and bacterial susceptibility to phagocytosis. Infect Immunol 30:370-374

15. Horan JD, English D, McPherson TA 1982 Association of neutrophil chemiluminescence with microbicidal activity. Clin Immunol Immunopathol 22:259-269

16. Smith CW, Hollers JC, Patrick RA, Hassett C 1979 Motility and adhesiveness in human neutrophils: effect of chemotactic factors. J Clin Invest 63:221229

17. Klein JO, Marcy SM 1990 Bacterial sepsis and meningitis. In: Remington JS, Klein JO (eds) Infectious Diseases of the Fetus and Newborn Infant. WB Saunders, Philadelphia, pp 601-656

18. Shigeoka AO, Santos JI, Hill HR 1979 Functional analysis of neutrophil granulocytes from healthy, infected and stressed neonates. J Pediatr 95:454460

19. Shigeoka AO, Charette RP, Wyman ML, Hill HR 1981 Defective oxidative metabolic responses of neutrophils from stressed neonates. J Pediatr 98:392398

20. Wright WCJ, Ank BJ, Herbert J, Steihm R 1975 Decreased bactericidal activity of leukocytes of stressed newborn infants. Pediatrics 56:579-584 